\title{
Mixing, Ergodic, and Nonergodic Processes with Rapidly Growing Information between Blocks
}

\author{
Łukasz Dębowski*
}

\begin{abstract}
We construct mixing processes over an infinite alphabet and ergodic processes over a finite alphabet for which Shannon mutual information between adjacent blocks of length $n$ grows as $n^{\beta}$, where $\beta \in(0,1)$. The processes are a modification of nonergodic Santa Fe processes, which were introduced in the context of natural language modeling. The rates of mutual information for the latter processes are alike and also established in this paper. As an auxiliary result, it is shown that infinite direct products of mixing processes are also mixing.
\end{abstract}

Key words: direct products, ergodic processes, mixing, mutual information, variable length coding

MSC 2010: 37A25, 94A 17

Running head: Processes with Rapidly Growing Information

${ }^{*}$ The research reported in Section $\amalg$ of this work was supported by the IST Programme of the European Community, under the PASCAL II Network of Excellence, IST-2002-506778.

Ł. Dębowski is with the Institute of Computer Science, Polish Academy of Sciences, ul. Ordona 21, 01-237 Warszawa, Poland (e-mail: ldebowsk@ipipan.waw.pl). 


\section{Introduction}

Let $H(X):=\mathbf{E}[-\log P(X)]$ denote the entropy of a discrete variable $X$ on a probability space $(\Omega, \mathcal{J}, P)$, where $\mathbf{E}$ is the expectation with respect to $P, \log$ is the binary logarithm, and the variable $P(X)$ takes the value $P(X=x)$ for $X=x$. We have the mutual information $I(X ; Y):=H(X)+H(Y)-H(X, Y)$ for finite entropies on the right hand side. Besides, we have the conditional entropy $H(X \mid Z)=H(X, Z)-H(Z)$ and the conditional mutual information $I(X ; Y \mid Z):=H(X \mid Z)+H(Y \mid Z)-H(X, Y \mid Z)$. These definitions are generalized to arbitrary random variables, e.g., in [1, 2].

Let $\left(X_{i}\right)_{i \in \mathbb{Z}}$ be a stationary process on $(\Omega, \mathcal{J}, P)$, where $X_{i}:(\Omega, \mathcal{J}) \rightarrow$ $(\mathbb{X}, \mathcal{X})$. For its distribution $\mu=P\left(\left(X_{i}\right)_{i \in \mathbb{Z}} \in \cdot\right)$ we denote the mutual information between blocks of length $n$ as

$$
E_{\mu}(n):=I\left(X_{1: n} ; X_{n+1: 2 n}\right) .
$$

The limiting value of mutual information, called excess entropy, is defined as

$$
E_{\mu}:=I\left(\left(X_{i}\right)_{i \leq 0} ;\left(X_{i}\right)_{i \geq 1}\right)=\lim _{n \rightarrow \infty} E_{\mu}(n)
$$

These quantities are natural measures of dependence for discrete-valued processes [3]. We are interested in constructing diverse examples of stationary measures for which

$$
E_{\mu}(n) \asymp n^{\beta},
$$

where $\beta \in(0,1)$, because certain measures of this kind may be useful for modeling natural language, cf., [4, 5].

Mentioning related results, let us first consider Gaussian processes. For theses processes the conditional mutual information equals $I\left(X_{0} ; X_{n} \mid\left(X_{i}\right)_{i=1}^{n-1}\right)=$ $-\log \left(1-|\alpha(n)|^{2}\right)$, where function $\alpha(k)$ is the partial autocorrelation, cf., $[6]$. Regardless of the alphabet, the mutual information between blocks may be reconstructed from conditional mutual information as

$$
E_{\mu}(n)=\sum_{k=1}^{n-1} k I\left(X_{0} ; X_{k} \mid\left(X_{i}\right)_{i=1}^{k-1}\right)+\sum_{k=n}^{2 n-1}(2 n-k) I\left(X_{0} ; X_{k} \mid\left(X_{i}\right)_{i=1}^{k-1}\right) .
$$

Thus the asymptotics (3) holds if and only if $\sum_{k=1}^{n} k|\alpha(k)|^{2} \asymp n^{\beta}$. As a result, the construction of processes that satisfy condition (3) is easy because the sole constraint on partial correlation reads $|\alpha(k)| \leq 1$ [7]. However, a classical result [8] says that excess entropy of nonsingular Gaussian autoregressive moving average (ARMA) processes is finite, cf., [3], [9, Theorem 9.4.1], [10, Section 5.5].

Some examples of stationary processes for which excess entropy is infinite are also known for discrete-valued processes. The trivial example for a countably infinite alphabet is a process such that $X_{i}$ does not depend on $i$ and $H\left(X_{i}\right)=\infty$. Then we have $E_{\mu}(n)=\infty$ for any $n \geq 1$. The aforementioned construction is impossible for processes over a finite alphabet. Considering those processes, we mention first that asymptotics $E(n)=(k / 2) \log (n / 2 \pi e)+O(1)$ holds for any Bayesian mixture of a $k$-parameter model with a prior concentrated on a subset of parameters with bounded Fisher information [11, Theorem 8.3]. Similar asymptotics $E(n) \asymp \log n$ holds for a binary process constructed by 
Gramss 12], cf., [13, 14]. The distribution of that process is formed by the frequencies of 0 's and 1's in the rabbit sequence. As for processes with infinite excess entropy that are mixing, Bradley [15] constructed a binary process which satisfies two conditions, cf., [16]: (i) the process is $\rho$-mixing and (ii) the restricted measure $P\left(\left(X_{i}\right)_{i \leq 0 \vee i \geq n} \in \cdot\right)$ is singular with respect to the product measure $P\left(\left(X_{i}\right)_{i<0} \in \cdot\right) \times P\left(\left(X_{i}\right)_{i}>n \in \cdot\right)$ for any $n \geq 1$ [15, Lemma 3]. The first property implies that the process is mixing in the ordinary ergodic theoretic sense 17, Volume 1, Chapters 3 and 5]. The second property implies that the excess entropy is infinite.

A few other examples concern hidden Markov chains. By the data processing inequality, excess entropy is finite for hidden Markov chains with a finite number of hidden states [18]. On the other hand, if the distribution of ergodic components of a stationary process has infinite entropy then the process has infinite excess entropy [2, Theorem 5]. Such a situation may arise for hidden Markov chains with a countably infinite number of hidden states. (Consider for instance a mixture of periodic processes where the probability of a period is a sufficiently slowly decreasing function of the cycle length 19].) A less trivial example, constructed in [19], is a stationary ergodic hidden Markov chain with infinite excess entropy, a finite number of output symbols, and a countably infinite alphabet of hidden states.

In this paper we will consider another class of processes that are nonergodic, ergodic, or mixing and satisfy condition (3). The construction of these processes is motivated linguistically. Let us first sketch this motivation. In our previous work 5], we have shown that proportionality (3) implies a power law which resembles Zipf's law for the distribution of words. Namely, product $E_{\mu}(n) \log n$ is upper bounded by the expected vocabulary size of an admissibly minimal grammar for the text of length $n$. It was empirically observed that the latter quantity approximates the number of distinct words for texts in natural language [20]. Our bound for mutual information and the vocabulary size holds if the alphabet $\mathbb{X}$ is finite and the process's distribution has finite energy property $[5$, Theorem 3]. There is also another linguistically motivated bound for $E_{\mu}(n)$. That one is a lower bound. Namely, asymptotics

$$
\limsup _{n \rightarrow \infty} E_{\mu}(n) / n^{\beta}>0
$$

follows from a hypothesis that texts describe an infinite random object in a highly repetitive way so that $n^{\beta}$ independent facts about the object can be inferred on average from the text of length $n[\underline{5}$, Theorem 2].

The goal of this paper is to prove the stronger asymptotics (3) for processes that were discussed in [5] and to define a new model of texts that describe a random object. So far, we have considered objects that do not change in time. This leads to models of texts being nonergodic measures. Here, we will admit objects that evolve slowly. That leads to models of texts which are mixing measures and still satisfy proportionality (3). In this way, linguistic inspiration contributes to better understanding of yet another problem in information theory.

Let us introduce our basic example. Throughout this paper, $\left(X_{i}\right)_{i \in \mathbb{Z}}$ denotes a stationary process on $(\Omega, \mathcal{J}, P)$ with $X_{i}:(\Omega, \mathcal{J}) \rightarrow(\mathbb{X}, \mathcal{X})$ and $\mathbb{X}=\mathbb{N} \times\{0,1\}$, where $\mathbb{N}$ is the set of positive integers. In the series of papers [2, 21, 5 ] we have examined some properties of the following process $\left(X_{i}\right)_{i \in \mathbb{Z}}$, called the (original) 
Santa Fe process in [5]. Namely, the variables $X_{i}$ consist of pairs

$$
X_{i}=\left(K_{i}, Z_{K_{i}}\right),
$$

where processes $\left(K_{i}\right)_{i \in \mathbb{Z}}$ and $\left(Z_{k}\right)_{k \in \mathbb{N}}$ are independent and distributed as follows. First, variables $Z_{k}$ are binary and equidistributed,

$$
P\left(Z_{k}=0\right)=P\left(Z_{k}=1\right)=1 / 2, \quad\left(Z_{k}\right)_{k \in \mathbb{N}} \sim \text { IID. }
$$

Second, variables $K_{i}$ obey the power law

$$
P\left(K_{i}=k\right)=k^{-1 / \beta} / \zeta\left(\beta^{-1}\right), \quad\left(K_{i}\right)_{i \in \mathbb{Z}} \sim \mathrm{IID},
$$

where $\beta \in(0,1)$ and $\zeta(x)=\sum_{k=1}^{\infty} k^{-x}$ is the zeta function.

Let us recall that $\mu=P\left(\left(X_{i}\right)_{i \in \mathbb{Z}} \in \cdot\right)$ and $E_{\mu}(n)=I\left(X_{1: n} ; X_{n+1: 2 n}\right)$. The first new result of this paper is:

Proposition 1 The block mutual information $E_{\mu}(n)$ for the original Santa Fe process $\left(X_{i}\right)_{i \in \mathbb{Z}}$ given by formula (6) obeys

$$
\lim _{n \rightarrow \infty} \frac{E_{\mu}(n)}{n^{\beta}}=\frac{\left(2-2^{\beta}\right) \Gamma(1-\beta)}{\left[\zeta\left(\beta^{-1}\right)\right]^{\beta}} .
$$

The calculation of the limit is facilitated by a decomposition of mutual information between blocks $X_{1: n}$ and $X_{n+1: 2 n}$ into a series of triple information among blocks $X_{1: n}$ and $X_{n+1: 2 n}$ and variables $Z_{k}$. This decomposition is a particular property of the Santa Fe process and some similar measures.

The uncommon construction of process (6) can be interpreted in this way. Imagine that the Santa Fe process is a sequence of statements which describe a random object $\left(Z_{k}\right)_{k \in \mathbb{N}}$ consistently. Each statement $X_{i}=(k, z)$ reveals both the address $k$ of a random bit of $\left(Z_{k}\right)_{k \in \mathbb{N}}$ and its value $Z_{k}=z$. Observe that the description is repetitive and consistent: if two statements $X_{i}=(k, z)$ and $X_{j}=\left(k^{\prime}, z^{\prime}\right)$ describe bits of the same address $\left(k=k^{\prime}\right)$ then they always assert the same bit value $\left(z=z^{\prime}\right)$. It follows hence that variables $Z_{k}$ can be predicted from realization $\left(X_{i}\right)_{i \in \mathbb{Z}}$ in a shift-invariant way and therefore the Santa Fe process is (strongly) nonergodic, cf., [2], [5, Definition 1].

Now let us introduce an example of a mixing process which satisfies (3). For this goal, we will replace individual variables $Z_{k}$ in the Santa Fe process with Markov chains $\left(Z_{i k}\right)_{i \in \mathbb{Z}}$. These Markov chains will be obtained by iterating a binary symmetric channel. Subsequently, the following process $\left(X_{i}\right)_{i \in \mathbb{Z}}$ will be called the generalized Santa Fe process. Let us put

$$
X_{i}=\left(K_{i}, Z_{i, K_{i}}\right),
$$

where processes $\left(K_{i}\right)_{i \in \mathbb{Z}}$ and $\left(Z_{i k}\right)_{i \in \mathbb{Z}}$, where $k \in \mathbb{N}$, are independent and distributed as follows. First, variables $K_{i}$ are distributed according to formula (8), as before. Second, each process $\left(Z_{i k}\right)_{i \in \mathbb{Z}}$ is a Markov chain with marginal distribution

$$
P\left(Z_{i k}=0\right)=P\left(Z_{i k}=1\right)=1 / 2
$$

and cross-over probabilities

$$
P\left(Z_{i k}=0 \mid Z_{i-1, k}=1\right)=P\left(Z_{i k}=1 \mid Z_{i-1, k}=0\right)=p_{k} .
$$


A linguistic interpretation of this process is as follows. Facts that are mentioned in texts repeatedly fall roughly under two types, as mentioned in the discussion of Definition 1 in [5]: (i) facts about objects that do not change in time (like mathematical or physical constants), and (ii) facts about objects that evolve with a varied speed (like culture, language, or geography). The random object $\left(Z_{k}\right)_{k \in \mathbb{N}}$ described by the original Santa Fe process does not evolve, or rather, no bit $Z_{k}$ is ever forgotten once revealed. On the other hand, the object $\left(Z_{i k}\right)_{k \in \mathbb{N}}$ described by the generalized Santa Fe process is a function of an instant $i$ and the probability that the $k$-th bit flips at a given instant equals $p_{k}$. For vanishing cross-over probabilities, the generalized Santa Fe process collapses to the original process.

As we will establish later in this paper, the generalized Santa Fe process is mixing for cross-over probabilities different to 0 or 1 .

Proposition 2 The generalized Santa Fe process $\left(X_{i}\right)_{i \in \mathbb{Z}}$ given by formula (10) is mixing for $p_{k} \in(0,1)$.

The proof consists in noticing that infinite direct products of mixing processes are mixing. This is an easy generalization of the well known fact for finite products [22, Chapter 10.§1].

We will also demonstrate this fact, which generalizes Proposition 1

Proposition 3 The block mutual information $E_{\mu}(n)$ for the generalized Santa Fe process $\left(X_{i}\right)_{i \in \mathbb{Z}}$ given by formula (10) obeys

$$
\limsup _{n \rightarrow \infty} \frac{E_{\mu}(n)}{n^{\beta}} \leq \frac{\left(2-2^{\beta}\right) \Gamma(1-\beta)}{\left[\zeta\left(\beta^{-1}\right)\right]^{\beta}} .
$$

The lower limits in particular cases are as follows:

(i) If $p_{k} \leq P\left(K_{i}=k\right)$ then

$$
\liminf _{n \rightarrow \infty} \frac{E_{\mu}(n)}{n^{\beta}} \geq \frac{A(\beta)}{\left[\zeta\left(\beta^{-1}\right)\right]^{\beta}},
$$

where

$$
A(\beta):=\sup _{\delta \in(1 / 2,1)}(1-\eta(\delta))^{\beta} \int_{\sqrt{\delta}}^{1} \frac{(1-u)^{2} d u}{u(-\ln u)^{\beta+1}}
$$

and $\eta(\delta)$ is the entropy of binary distribution $(\delta, 1-\delta)$,

$$
\eta(\delta):=-\delta \log \delta-(1-\delta) \log (1-\delta) .
$$

(ii) If $\lim _{k \rightarrow \infty} p_{k} / P\left(K_{i}=k\right)=0$ then $E_{\mu}(n)$ obeys (9)).

Now let us introduce a similar ergodic process over a finite alphabet. For this goal we use a transformation of processes over an infinite alphabet into processes over a finite alphabet that preserves stationarity and (non)ergodicity and does not distort entropy too much, as we have shown in [21]. We call this transformation stationary (variable-length) coding. (The same or a similar construction has been considered in [23, 24, 25].) It is a composition of two operations. 
First, let a function $f: \mathbb{X} \rightarrow \mathbb{Y}^{*}$, called a coding function, map symbols from alphabet $\mathbb{X}$ into strings over another alphabet $\mathbb{Y}$. We define its extension to double infinite sequences $f^{\mathbb{Z}}: \mathbb{X}^{\mathbb{Z}} \rightarrow \mathbb{Y}^{\mathbb{Z}} \cup\left(\mathbb{Y}^{*} \times \mathbb{Y}^{*}\right)$ as

$$
f^{\mathbb{Z}}\left(\left(x_{i}\right)_{i \in \mathbb{Z}}\right):=\ldots f\left(x_{-1}\right) f\left(x_{0}\right) \cdot f\left(x_{1}\right) f\left(x_{2}\right) \ldots
$$

where $x_{i} \in \mathbb{X}$ and the bold-face dot separates the 0 -th and the first symbol. Then for a stationary process $\left(X_{i}\right)_{i \in \mathbb{Z}}$ on $(\Omega, \mathcal{J}, P)$, where variables $X_{i}$ take values in space $(\mathbb{X}, \mathcal{X})$, we introduce process

$$
\left(Y_{i}\right)_{i \in \mathbb{Z}}:=f^{\mathbb{Z}}\left(\left(X_{i}\right)_{i \in \mathbb{Z}}\right)
$$

where variables $Y_{i}$ take values in space $(\mathbb{Y}, \mathcal{Y})$, as long as the right hand side is a double infinite sequence almost surely.

The second operation is as follows. Transformation (17) does not preserve stationarity in general but process $\left(Y_{i}\right)_{i \in \mathbb{Z}}$ is asymptotically mean stationary (AMS) under mild conditions [21, Proposition 2.3], which are satisfied in the setting considered further. Then for the distribution

$$
P\left(\left(Y_{i}\right)_{i \in \mathbb{Z}} \in \cdot\right)=\nu
$$

and the shift operation $T\left(\left(y_{i}\right)_{i \in \mathbb{Z}}\right):=\left(y_{i+1}\right)_{i \in \mathbb{Z}}$ there exists a stationary measure

$$
\bar{\nu}(A):=\lim _{n \rightarrow \infty} \frac{1}{n} \sum_{i=0}^{n-1} \nu \circ T^{-i}(A),
$$

called the stationary mean of $\nu[24,21]$. It is convenient to suppose that probability space $(\Omega, \mathcal{J}, P)$ is rich enough to support a process $\left(\bar{Y}_{i}\right)_{i \in \mathbb{Z}}$ with the distribution

$$
P\left(\left(\bar{Y}_{i}\right)_{i \in \mathbb{Z}} \in \cdot\right)=\bar{\nu}
$$

Whereas process $\left(Y_{i}\right)_{i \in \mathbb{Z}}$ need not be stationary, process $\left(\bar{Y}_{i}\right)_{i \in \mathbb{Z}}$ is stationary and will be called the stationary (variable-length) coding of $\left(X_{i}\right)_{i \in \mathbb{Z} \text {. }}$

Processes $\left(X_{i}\right)_{i \in \mathbb{Z}},\left(Y_{i}\right)_{i \in \mathbb{Z}}$, and $\left(\bar{Y}_{i}\right)_{i \in \mathbb{Z}}$ have isomorphic shift-invariant algebras for some nice coding functions, called synchronizable injections [21, Proposition 3.3]. For example, for the infinite alphabet $\mathbb{X}=\mathbb{N} \times\{0,1\}$, let us assume the ternary alphabet $\mathbb{Y}=\{0,1,2\}$ and the coding function

$$
f(k, z)=b(k) z 2,
$$

where $b(k) \in\{0,1\}^{+}$is the binary representation of a natural number $k$ stripped of the leading digit 1. Coding function (21) is an instance of a synchronizable injection. Hence we have the following fact:

Proposition 4 Let $\left(\bar{Y}_{i}\right)_{i \in \mathbb{Z}}$ be the stationary coding obtained from applying the coding function (21) to the generalized Santa Fe process (10). Process $\left(\bar{Y}_{i}\right)_{i \in \mathbb{Z}}$ is nonergodic if $p_{k}=0$ and ergodic if $p_{k} \in(0,1)$.

Notice, however, that the stationary coding of a mixing process is not mixing for a synchronizable coding function in general. For example, if we take the generalized Santa Fe process and the coding function $f(k, z)=01$, which is also 
a synchronizable injection, the stationary coding $\left(\bar{Y}_{i}\right)_{i \in \mathbb{Z}}$ is not mixing because of periodic oscillations in the realizations of the process $\left(Y_{i}\right)_{i \in \mathbb{Z}}$. Such regular periods do not arise for the generalized Santa Fe process and the coding function (21) since variables $\left|f\left(X_{i}\right)\right|$, where $|w|$ is the length of string $w$, differ from constants and are independent and identically distributed. Thus, we conjecture that the resulted process $\left(\bar{Y}_{i}\right)_{i \in \mathbb{Z}}$ is mixing for $p_{k} \in(0,1)$.

Now let us consider block mutual information for the stationary coding of the generalized Santa Fe process. Let us recall that $\bar{\nu}=P\left(\left(\bar{Y}_{i}\right)_{i \in \mathbb{Z}} \in \cdot\right)$ and $E_{\bar{\nu}}(m)=I\left(\bar{Y}_{1: m} ; \bar{Y}_{m+1: 2 m}\right)$. As the last new result, we will show this fact:

Proposition 5 Let $\left(\bar{Y}_{i}\right)_{i \in \mathbb{Z}}$ be the stationary coding obtained from applying the coding function (21) to the generalized Santa Fe process (10). Define the expansion rate $L:=\mathbf{E}\left|f\left(X_{i}\right)\right|$. The block mutual information $E_{\bar{\nu}}(m)$ for process $\left(\bar{Y}_{i}\right)_{i \in \mathbb{Z}}$ satisfies

$$
\limsup _{m \rightarrow \infty} \frac{E_{\bar{\nu}}(m)}{m^{\beta}} \leq \frac{1}{L^{\beta}} \frac{\left(2-2^{\beta}\right) \Gamma(1-\beta)}{\left[\zeta\left(\beta^{-1}\right)\right]^{\beta}} .
$$

The lower limits in particular cases are as follows:

(i) If $p_{k} \leq P\left(K_{i}=k\right)$ then

$$
\liminf _{m \rightarrow \infty} \frac{E_{\bar{\nu}}(m)}{m^{\beta}} \geq \frac{1}{L^{\beta}} \frac{A(\beta)}{\left[\zeta\left(\beta^{-1}\right)\right]^{\beta}} .
$$

where $A(\beta)$ is defined in (15).

(ii) If $\lim _{k \rightarrow \infty} p_{k} / P\left(K_{i}=k\right)=0$ then

$$
\lim _{m \rightarrow \infty} \frac{E_{\bar{\nu}}(m)}{m^{\beta}}=\frac{1}{L^{\beta}} \frac{\left(2-2^{\beta}\right) \Gamma(1-\beta)}{\left[\zeta\left(\beta^{-1}\right)\right]^{\beta}} .
$$

Proposition 5 follows from Proposition 3 by the conditional data processing inequality and Chernoff bounds. This proposition strengthens inequality

$$
\limsup _{m \rightarrow \infty} \frac{E_{\bar{\nu}}(m)}{m^{\beta}}>0,
$$

which follows for $p_{k}=0$ by [21, Proposition 1.4] and [5, Theorem 2].

The further organization of this paper is as follows. The rate of mutual information for the original and generalized Santa Fe processes is discussed in Section II. The rate of mutual information for the stationary coding is established in Section III. Subsequently, the mixing property for the generalized Santa Fe process is shown in Appendix A. As an auxiliary result, we demonstrate that infinite direct products of mixing processes are also mixing.

\section{The rate of mutual information}

In this section we evaluate the rate of block mutual information for the Santa Fe process and its mixing counterpart. The main tool is conditional mutual information for stochastic processes as discussed, e.g., in [1, 2].

Here are some facts about conditional information that will be used, cf., [2]: 
(a) continuity $I\left(X ;\left(Y_{k}\right)_{k \in \mathbb{N}} \mid Z\right)=\lim _{n \rightarrow \infty} I\left(X ;\left(Y_{k}\right)_{k=1}^{n} \mid Z\right)$,

(b) chain rule $I(X ; Y, Z \mid W)=I(X ; Y \mid W)+I(X ; Z \mid Y, W)$, and

(c) equality $I(X ; Y \mid Z)=0$ for $X$ and $Y$ conditionally independent given $Z$.

Two simple corollaries of the chain rule will be used as well:

(i) $I(X ; Y)=I(X ; Y ; Z)+I(X ; Y \mid Z)$ for $H(X), H(Y)<\infty$, where we define triple information

$$
I(X ; Y ; Z):=I(X ; Z)+I(Y ; Z)-I((X, Y) ; Z),
$$

(ii) $I(X ; Z \mid Y)=I(X ; Z)$ for $X$ and $Y$ independent and conditionally independent given $Z$.

The second identity follows from $I(X ;(Y, Z))=I(X ; Y)+I(X ; Z \mid Y)=$ $I(X ; Z)+I(X ; Y \mid Z)$ where both $I(X ; Y)=0$ and $I(X ; Y \mid Z)=0$.

Now we can evaluate block mutual information $E_{\mu}(n)$ for the Santa Fe processes. The case of the original Santa Fe process is simpler and will be considered separately to guide the reader through the more complicated proof for the generalized process.

Proof of Proposition 1; Notice that variables $Z_{k}, k \in \mathbb{N}$, are independent and conditionally independent given any finite block $X_{n: m}$. Hence

$$
I\left(X_{1: n} ;\left(Z_{k}\right)_{k \in \mathbb{N}}\right)=\sum_{k=1}^{\infty} I\left(X_{1: n} ; Z_{k} \mid Z_{1: k-1}\right)=\sum_{k=1}^{\infty} I\left(X_{1: n} ; Z_{k}\right) .
$$

Also $X_{1: n}$ and $X_{n+1: 2 n}$ are conditionally independent given $\left(Z_{k}\right)_{k \in \mathbb{N}}$. Hence $I\left(X_{1: n} ; X_{n+1: 2 n} \mid\left(Z_{k}\right)_{k \in \mathbb{N}}\right)=0$. Both results yield

$$
\begin{aligned}
E_{\mu}(n) & =I\left(X_{1: n} ; X_{n+1: 2 n}\right) \\
& =I\left(X_{1: n} ; X_{n+1: 2 n} ;\left(Z_{k}\right)_{k \in \mathbb{N}}\right)+I\left(X_{1: n} ; X_{n+1: 2 n} \mid\left(Z_{k}\right)_{k \in \mathbb{N}}\right) \\
& =I\left(X_{1: n} ; X_{n+1: 2 n} ;\left(Z_{k}\right)_{k \in \mathbb{N}}\right) \\
& =2 I\left(X_{1: n} ;\left(Z_{k}\right)_{k \in \mathbb{N}}\right)-I\left(X_{1: 2 n} ;\left(Z_{k}\right)_{k \in \mathbb{N}}\right) \\
& =\sum_{k=1}^{\infty}\left[2 I\left(X_{1: n} ; Z_{k}\right)-I\left(X_{1: 2 n} ; Z_{k}\right)\right] \\
& =\sum_{k=1}^{\infty} I\left(X_{1: n} ; X_{n+1: 2 n} ; Z_{k}\right) .
\end{aligned}
$$

Computing simple expressions

$$
\begin{aligned}
H\left(Z_{k} \mid X_{1: n}\right)= & 1 \cdot P\left(K_{i} \neq k \text { for all } i \in\{1, \ldots, n\}\right) \\
& +0 \cdot P\left(K_{i}=k \text { for some } i \in\{1, \ldots, n\}\right), \\
I\left(X_{1: n} ; Z_{k}\right)= & P\left(K_{i}=k \text { for some } i \in\{1, \ldots, n\}\right) \\
= & \left(1-\left[1-P\left(K_{i}=k\right)\right]^{n}\right),
\end{aligned}
$$

we obtain the triple mutual information

$$
I\left(X_{1: n} ; X_{n+1: 2 n} ; Z_{k}\right)=\left(1-\left[1-P\left(K_{i}=k\right)\right]^{n}\right)^{2}
$$


and the block mutual information

$$
E_{\mu}(n)=\sum_{k=1}^{\infty}\left(1-\left(1-\frac{A}{k^{1 / \beta}}\right)^{n}\right)^{2},
$$

where $A:=1 / \zeta\left(\beta^{-1}\right)$.

The right-hand side of (27) equals up to an additive constant $\leq 1$ to the integral

$$
\int_{1}^{\infty}\left(1-\left(1-\frac{A}{k^{1 / \beta}}\right)^{n}\right)^{2} d k=\beta(A n)^{\beta} \int_{(1-A)^{n}}^{1} f_{n}(u) d u
$$

where we use substitution

$$
u:=\left(1-A k^{-1 / \beta}\right)^{n}
$$

and functions

$$
f_{n}(u):=\frac{(1-u)^{2}}{u^{1-1 / n}\left[n\left(1-u^{1 / n}\right)\right]^{\beta+1}} .
$$

We have the limit

$$
\lim _{n \rightarrow \infty} f_{n}(u)=f(u):=\frac{(1-u)^{2}}{u(-\ln u)^{\beta+1}}
$$

with the upper bound

$$
\frac{f_{n}(u)}{f(u)} \leq \sup _{0<x<1} \frac{x(-\ln x)^{\beta+1}}{(1-x)^{\beta+1}}=1, \quad u, \beta \in(0,1) .
$$

Moreover, function $f(u)$ is integrable on $u \in(0,1)$. Hence

$$
\lim _{n \rightarrow \infty} \frac{E_{\mu}(n)}{n^{\beta}}=\beta A^{\beta} \int_{0}^{1} f(u) d u
$$

follows by the dominated convergence theorem.

It remains to compute $\int f(u) d u$. Putting $t:=-\ln u$ yields

$$
\begin{aligned}
\int_{0}^{1} f(u) d u & =\int_{0}^{\infty}\left(1-e^{-t}\right)^{2} t^{-\beta-1} d t \\
& =\int_{0}^{\infty}\left[e^{-2 t}-1-2\left(e^{-t}-1\right)\right] t^{-\beta-1} d t \\
& =\left(2-2^{\beta}\right) \beta^{-1} \Gamma(1-\beta),
\end{aligned}
$$

where integral

$$
\begin{aligned}
\int_{0}^{\infty} & \left(e^{-k t}-1\right) t^{-\beta-1} d t=\left.\left(e^{-k t}-1\right)\left(-\beta^{-1}\right) t^{-\beta}\right|_{0} ^{\infty} \\
& -\int_{0}^{\infty}\left(-k e^{-k t}\right)\left(-\beta^{-1}\right) t^{-\beta} d t=-k^{\beta} \beta^{-1} \Gamma(1-\beta)
\end{aligned}
$$

can be integrated by parts for the considered $\beta$. 
Next, we prove the more general statement, partly using the preceding proof.

Proof of Proposition 3, Observe that processes $\tilde{Z}_{k}:=\left(Z_{i k}\right)_{i \in \mathbb{Z}}$, where $k \in \mathbb{N}$, are independent and conditionally independent given any finite block $X_{n: m}$. Also $X_{1: n}$ and $X_{n+1: 2 n}$ are conditionally independent given $\left(\tilde{Z}_{k}\right)_{k \in \mathbb{N}}$. Thus we obtain

$$
E_{\mu}(n)=\sum_{k=1}^{\infty} I\left(X_{1: n} ; X_{n+1: 2 n} ; \tilde{Z}_{k}\right)
$$

by replacing $Z_{k}$ with $\tilde{Z}_{k}$ in derivation (26) from the previous proof.

By the assumed Markov property, process $\tilde{Z}_{k}=\left(Z_{i k}\right)_{i \in \mathbb{Z}}$ is independent from $X_{1: n}$ given $\left(Z_{i k}\right)_{1 \leq i \leq n}$. This yields

$$
I\left(X_{1: n} ; X_{n+1: 2 n} ; \tilde{Z}_{k}\right)=2 I\left(X_{1: n} ;\left(Z_{i k}\right)_{1 \leq i \leq n}\right)-I\left(X_{1: 2 n} ;\left(Z_{i k}\right)_{1 \leq i \leq 2 n}\right) .
$$

The expressions on the right-hand side can be analyzed as

$$
I\left(X_{1: n} ;\left(Z_{i k}\right)_{1 \leq i \leq n}\right)=\sum_{i=1}^{n} I\left(X_{i} ; Z_{i k} \mid X_{1: i-1}\right)
$$

because $\left(Z_{i k}\right)_{1 \leq i \leq n}$ is independent from $X_{i}$ given $Z_{i k}$ and $X_{1: i-1}$. Moreover,

$$
I\left(X_{i} ; Z_{i k} \mid X_{1: i-1}\right)=H\left(Z_{i k} \mid X_{1: i-1}\right)-H\left(Z_{i k} \mid X_{1: i}\right) .
$$

To evaluate the conditional entropies, put $a_{n k}:=\eta\left(P\left(Z_{i k}=z \mid Z_{i-n, k}=z\right)\right)$ and $b_{k}:=P\left(K_{i}=k\right)$. Notice that by the Markovity of $\left(Z_{i k}\right)_{i \in \mathbb{Z}}$ we have

$$
\begin{aligned}
H\left(Z_{i k} \mid X_{1: i-1}\right)= & \sum_{n=1}^{i-1} a_{n k} P\left(K_{j} \neq k \text { for } i-n<j \leq i-1\right) P\left(K_{i-n}=k\right) \\
& +\eta\left(P\left(Z_{i k}=z\right)\right) P\left(K_{j} \neq k \text { for } 1 \leq j \leq i-1\right) \\
= & \sum_{n=1}^{i-1} a_{n k} b_{k}\left(1-b_{k}\right)^{n-1}+\left(1-b_{k}\right)^{i-1} .
\end{aligned}
$$

Similarly, since $a_{0 k}=0$, we obtain

$$
\begin{aligned}
H\left(Z_{i k} \mid X_{1: i}\right)= & \sum_{n=0}^{i-1} a_{n k} P\left(K_{j} \neq k \text { for } i-n<j \leq i\right) P\left(K_{i-n}=k\right) \\
& +\eta\left(P\left(Z_{i k}=z\right)\right) P\left(K_{j} \neq k \text { for } 1 \leq j \leq i\right) \\
= & \sum_{n=1}^{i-1} a_{n k} b_{k}\left(1-b_{k}\right)^{n}+\left(1-b_{k}\right)^{i} .
\end{aligned}
$$

Thus we may reconstruct

$$
\begin{aligned}
I\left(X_{i} ; Z_{i k} \mid X_{1: i-1}\right) & =\sum_{n=1}^{i-1} a_{n k} b_{k}^{2}\left(1-b_{k}\right)^{n-1}+\left[\left(1-b_{k}\right)^{i-1}-\left(1-b_{k}\right)^{i}\right], \\
I\left(X_{1: n} ;\left(Z_{i k}\right)_{1 \leq i \leq n}\right) & =\sum_{m=1}^{n-1}(n-m) a_{m k} b_{k}^{2}\left(1-b_{k}\right)^{m-1}+\left[1-\left(1-b_{k}\right)^{n}\right],
\end{aligned}
$$


and

$$
\begin{aligned}
I\left(X_{1: n} ; X_{n+1: 2 n} ; \tilde{Z}_{k}\right)= & -\sum_{m=1}^{n-1} m a_{m k} b_{k}^{2}\left(1-b_{k}\right)^{m-1} \\
& -\sum_{m=n}^{2 n-1}(2 n-m) a_{m k} b_{k}^{2}\left(1-b_{k}\right)^{m-1}+\left[1-\left(1-b_{k}\right)^{n}\right]^{2} .
\end{aligned}
$$

For a fixed $b_{k}$, we see that $I\left(X_{1: n} ; X_{n+1: 2 n} ; \tilde{Z}_{k}\right)$ is minimized for $a_{m k}=1$. This case arises when $p_{k}=1 / 2$ and $\left(Z_{i k}\right)_{i \in \mathbb{Z}}$ are IID. A direct evaluation yields then $H\left(Z_{i k} \mid X_{1: i-1}\right)=1, H\left(Z_{i k} \mid X_{1: i}\right)=\left(1-b_{k}\right), I\left(X_{1: n} ;\left(Z_{i k}\right)_{1 \leq i \leq n}\right)=n b_{k}$, and $I\left(X_{1: n} ; X_{n+1: 2 n} ; \tilde{Z}_{k}\right)=0$. In this way we have proved that

$$
\sum_{m=1}^{n-1} m b_{k}^{2}\left(1-b_{k}\right)^{m-1}+\sum_{m=n}^{2 n-1}(2 n-m) b_{k}^{2}\left(1-b_{k}\right)^{m-1}=\left[1-\left(1-b_{k}\right)^{n}\right]^{2} .
$$

On the other hand, $I\left(X_{1: n} ; X_{n+1: 2 n} ; \tilde{Z}_{k}\right)$ is maximized for $a_{m k}=0$. This holds if $p_{k}=0$ or $p_{k}=1$. For $p_{k}=0$, the process $\left(X_{i}\right)_{i \in \mathbb{Z}}$ collapses to (6).

By equality (30), we obtain

$$
I\left(X_{1: n} ; X_{n+1: 2 n} ; \tilde{Z}_{k}\right) \in\left[(1-\epsilon)\left[1-\left(1-b_{k}\right)^{n}\right]^{2},\left[1-\left(1-b_{k}\right)^{n}\right]^{2}\right]
$$

if $a_{m k} \leq \epsilon$ for $m \leq 2 n-1$. To bound coefficients $a_{m k}$, observe

$$
P\left(Z_{i k}=z \mid Z_{i-n, k}=z\right) \geq\left(1-p_{k}\right)^{n} .
$$

Hence $a_{m k} \leq \eta(\delta)$ for $m \leq 2 n-1$ if $\left(1-p_{k}\right)^{2 n} \geq \delta \geq 1 / 2$. Thus we obtain

$$
E_{\mu}(n) \in\left[(1-\eta(\delta)) \sum_{k \in \mathbb{N}:\left(1-p_{k}\right)^{2 n} \geq \delta}\left[1-\left(1-b_{k}\right)^{n}\right]^{2}, \sum_{k \in \mathbb{N}}\left[1-\left(1-b_{k}\right)^{n}\right]^{2}\right] \text {. }
$$

The most tedious part of the proof is completed.

The limiting behavior of the upper bound in (31) has been analyzed in the proof of Proposition 1, and by that reasoning (13) holds. Now we will consider the limit of the lower bound in (31). As in the previous proof, we will approximate the respective sum with an integral. Recall that $b_{k}=A k^{-1 / \beta}$ with $A=1 / \zeta\left(\beta^{-1}\right)$. Let us define $b_{k}$ for real $k$ in the same way.

(i) For $p_{k} \leq b_{k}$ : notice that $\left(1-b_{k}\right)^{2 n} \geq \delta$ implies $\left(1-p_{k}\right)^{2 n} \geq \delta$. Thus $E_{\mu}(n) /(1-\eta(\delta))+1$ is greater than

$$
\int_{\left(1-b_{k}\right)^{n} \geq \sqrt{\delta}}^{\infty}\left(1-\left(1-b_{k}\right)^{n}\right)^{2} d k=\beta(A n)^{\beta} \int_{\sqrt{\delta}}^{1} f_{n}(u) d u,
$$

where we use substitution (28) and functions (29). This yields (14) by the dominated convergence theorem. 
(ii) For $\lim _{k} p_{k} / b_{k}=0$ : let $k(n)$ be the largest number $k$ such that $\left(1-p_{k}\right)^{2 n}<$ $\delta$ or put $k(n)=1$ if there is no such number. Then $E_{\mu}(n) /(1-\eta(\delta))+1$ is greater than

$$
\int_{k(n)}^{\infty}\left(1-\left(1-b_{k}\right)^{n}\right)^{2} d k=\beta(A n)^{\beta} \int_{u(n)}^{1} f_{n}(u) d u
$$

where $u(n):=\left(1-b_{k(n)}\right)^{n}$. We have $\lim _{n} u(n)=0$ if $\lim _{n} k(n)<\infty$. On the other hand, if $\lim _{n} k(n)=\infty$ then we use $\liminf n_{n} n p_{k(n)}>-\ln \sqrt{\delta}$ and $\lim _{k} p_{k} / b_{k}=0$ to infer $\lim _{n} n b_{k(n)}=\infty$ and hence $\lim _{n} u(n)=0$. Thus the dominated convergence theorem in both cases yields

$$
\liminf _{n \rightarrow \infty} \frac{E_{\mu}(n)}{n^{\beta}} \geq(1-\eta(\delta)) \beta A^{\beta} \int_{0}^{1} f(u) d u .
$$

Taking $\delta \rightarrow 1$ gives (9).

\section{Encoding into a finite alphabet}

In this section we study the rate of mutual information for the stationary coding of the generalized Santa Fe process. Let $|w|$ be the length of string $w$ and let $\left(X_{i}\right)_{i \in \mathbb{Z}}$ denote the generalized Santa Fe process. For the coding function (21), regardless of the value of $p_{k}$, the expansion rate

$$
\lim _{n \rightarrow \infty} \frac{1}{n} \sum_{i=1}^{n}\left|f\left(X_{i}\right)\right|
$$

is almost surely constant and equals the expansion rate $L:=\mathbf{E}\left|f\left(X_{i}\right)\right|$. Hence the stationary coding $\left(\bar{Y}_{i}\right)_{i \in \mathbb{Z}}$ can be constructed as detailed below. This construction was formally introduced in [21, Section 6] and justified by [21, Proposition 2.3].

Suppose that probability space $(\Omega, \mathfrak{J}, P)$ is sufficiently rich to support some previously unmentioned random variable $N: \Omega \rightarrow \mathbb{N} \cup\{0\}$, called a random shift, and a nonstationary process $\left(\bar{X}_{i}\right)_{i \in \mathbb{Z}}$ where $\bar{X}_{i}: \Omega \rightarrow \mathbb{X}$. We assume that $N$ and $\left(\bar{X}_{i}\right)_{i \in \mathbb{Z}}$ are conditionally independent given $\bar{X}_{0}$ and their distribution is

$$
\begin{array}{cc}
P\left(\bar{X}_{k: l}=x_{k: l}\right)=P\left(X_{k: l}=x_{k: l}\right) \cdot \frac{\left|f\left(x_{0}\right)\right|}{L}, & k \leq 0 \leq l, \\
P\left(N=n \mid \bar{X}_{0}=x_{0}\right)=\frac{\mathbf{1}_{\left\{0 \leq n \leq\left|f\left(x_{0}\right)\right|-1\right\}}}{\left|f\left(x_{0}\right)\right|}, & n \in \mathbb{N} \cup\{0\} .
\end{array}
$$

Process $\left(\bar{Y}_{i}\right)_{i \in \mathbb{Z}}$ with the desired distribution $\bar{\nu}=P\left(\left(\bar{Y}_{i}\right)_{i \in \mathbb{Z}} \in \cdot\right)$, where $\nu=$ $P\left(\left(Y_{i}\right)_{i \in \mathbb{Z}} \in \cdot\right)$ for $\left(Y_{i}\right)_{i \in \mathbb{Z}}=f^{\mathbb{Z}}\left(\left(X_{i}\right)_{i \in \mathbb{Z}}\right)$, can be obtained as

$$
\left(\bar{Y}_{i}\right)_{i \in \mathbb{Z}}=T^{-N} f^{\mathbb{Z}}\left(\left(\bar{X}_{i}\right)_{i \in \mathbb{Z}}\right),
$$

where $T\left(\left(y_{i}\right)_{i \in \mathbb{Z}}\right):=\left(y_{i+1}\right)_{i \in \mathbb{Z}}$ is the shift operation.

Lemma 1 Denote blocks $\bar{X}_{k: l}$ with $\bar{X}_{0}$ removed as $\bar{X}_{k: l \backslash 0}$. For the Santa Fe processes variables $\bar{X}_{k: l \backslash 0}$ and $X_{k: l \backslash 0}$ have the same distribution. 
Proof: Notice that $\left|f\left(X_{0}\right)\right|$ does not depend on $Z_{0, K_{0}}$ and $K_{0}$ is independent of $X_{k: l \backslash 0}$. Hence

$$
\begin{aligned}
P\left(\bar{X}_{k: l \backslash 0}\right) & =P\left(X_{k: l \backslash 0}\right) \sum_{x_{0}} \frac{\left|f\left(x_{0}\right)\right|}{L} P\left(X_{0}=x_{0} \mid X_{k: l \backslash 0}\right) \\
& =P\left(X_{k: l \backslash 0}\right) \sum_{k_{0}, z_{0}} \frac{\left|f\left(k_{0}, 1\right)\right|}{L} P\left(K_{0}=k_{0}\right) P\left(Z_{0, k_{0}}=z_{0} \mid X_{k: l \backslash 0}\right) \\
& =P\left(X_{k: l \backslash 0}\right) .
\end{aligned}
$$

In the following we write $L_{i}:=\left|f\left(X_{i}\right)\right|$ and $\bar{L}_{i}:=\left|f\left(\bar{X}_{i}\right)\right|$. Variables $L_{i}$ are independent and identically distributed. For these variables we define indices

$$
\begin{aligned}
L_{t}^{+} & :=\frac{1}{t} \log \mathbf{E} 2^{t L_{i}}, \\
L_{t}^{-} & :=-\frac{1}{t} \log \mathbf{E} 2^{-t L_{i}},
\end{aligned}
$$

where $t>0$. For the given distribution of $L_{i}$, we have $0<L_{t}^{-}, L_{t}^{+}<\infty$ for sufficiently small $t$. By the Jensen inequality $L_{t}^{+}$is a growing function of $t$ and $L_{t}^{-}$is a decreasing function of $t$. Jensen inequality implies also $L_{t}^{-} \leq L \leq L_{t}^{+}$.

Lemma 2 We have

$$
\lim _{t \rightarrow 0} L_{t}^{+}=\lim _{t \rightarrow 0} L_{t}^{-}=L .
$$

Proof: Consider function $g(t, x)=t^{-2}\left(2^{t x}-1-t x\right)$. For $x>0$, it is a growing function of $t$. Consider next such a $t_{0}$ that $\mathbf{E} 2^{t_{0} L_{i}}<\infty$. For $0<t \leq t_{0}$, we obtain

$$
\mathbf{E} 2^{t L_{i}}=1+t \mathbf{E} L_{i}+t^{2} \mathbf{E} g\left(t, L_{i}\right) \leq 1+t \mathbf{E} L_{i}+t^{2} \mathbf{E} g\left(t_{0}, L_{i}\right) .
$$

This yields

$$
L \leq L_{t}^{+} \leq \frac{1}{t} \log \left(1+t \mathbf{E} L_{i}+t^{2} \mathbf{E} g\left(t_{0}, L_{i}\right)\right) \stackrel{t \rightarrow 0}{\longrightarrow} L .
$$

On the other hand, for $t>0$, we have

$$
\mathbf{E} 2^{-t L_{i}} \leq 1-t \mathbf{E} L_{i}+\frac{t^{2} \mathbf{E} L_{i}^{2}}{2}
$$

Hence

$$
L \geq L_{t}^{-} \geq-\frac{1}{t} \log \left(1-t \mathbf{E} L_{i}+\frac{t^{2} \mathbf{E} L_{i}^{2}}{2}\right) \stackrel{t \rightarrow 0}{\longrightarrow} L .
$$


Define events

$$
\begin{aligned}
S_{n}^{+} & :=\left(\sum_{i=1}^{n} L_{i}<n\left(L_{t}^{+}+\epsilon\right)\right), \\
S_{n}^{-} & :=\left(\sum_{i=1}^{n} L_{i}>n\left(L_{t}^{-}-\epsilon\right)\right), \\
T_{n}^{+} & :=\left(\sum_{i=-n+1}^{-1} L_{i}<(n-1)\left(L_{t}^{+}+\epsilon\right)\right), \\
T_{n}^{-} & :=\left(\sum_{i=-n+1}^{-1} L_{i}>(n-1)\left(L_{t}^{-}-\epsilon\right)\right) .
\end{aligned}
$$

Subsequently, we will use the Chernoff bounds:

Lemma 3 For $t>0$ and $\epsilon>0$,

$$
\begin{aligned}
& P\left(S_{n}^{+^{c}}\right) \leq \frac{1}{2^{n t \epsilon}}, \\
& P\left(S_{n}^{-c}\right) \leq \frac{1}{2^{n t \epsilon}}, \\
& P\left(T_{n}^{+c}\right) \leq \frac{1}{2^{(n-1) t \epsilon}}, \\
& P\left(T_{n}^{-c}\right) \leq \frac{1}{2^{(n-1) t \epsilon}} .
\end{aligned}
$$

Proof: Because variables $L_{i}$ are independent and identically distributed, using Markov inequality we observe

$$
\begin{aligned}
& P\left(S_{n}^{+^{c}}\right)=P\left(2^{t \sum_{i} L_{i}} \geq 2^{t n\left(L_{t}^{+}+\epsilon\right)}\right) \leq \frac{\mathbf{E} 2^{t \sum_{i} L_{i}}}{2^{\operatorname{tn}\left(L_{t}^{+}+\epsilon\right)}} \leq \frac{1}{2^{n t \epsilon}} \\
& P\left(S_{n}^{-c}\right)=P\left(2^{-t \sum_{i} L_{i}} \geq 2^{-t n\left(L_{t}^{-}-\epsilon\right)}\right) \leq \frac{\mathbf{E} 2^{-t \sum_{i} L_{i}}}{2^{-\operatorname{tn}\left(L_{t}^{-}-\epsilon\right)}} \leq \frac{1}{2^{n t \epsilon}} .
\end{aligned}
$$

Analogously we obtain the claims for $T_{n}^{+^{c}}$ and $T_{n}^{-c}$.

Next, for an event $E$, we introduce conditional entropy $H(X \mid E)$ and mutual information $I(X ; Y \mid E)$ which are respectively the entropy of variable $X$ and mutual information between variables $X$ and $Y$ taken with respect to probability measure $P(\cdot \mid E)$.

Lemma 4 For the generalized Santa Fe process, let $\gamma:=\beta /(1-\beta)$ and $s<$ $\min (t / 2, \gamma / 2)$. Then for sufficiently large $n$,

$$
\begin{gathered}
P\left(S_{n}^{+^{c}}\right) H\left(L_{1} \mid{S_{n}^{+}}^{c}\right) \leq \frac{1}{2^{n s \epsilon}}, \\
P\left(T_{n}^{+^{c}}\right) H\left(L_{-1} \mid T_{n}^{+^{c}}\right) \leq \frac{1}{2^{(n-1) s \epsilon}} .
\end{gathered}
$$

Proof: We have

$$
P\left(L_{i}=l\right)=\sum_{k=2^{l-1}}^{2^{l}-1} \frac{k^{-1 / \beta}}{\zeta\left(\beta^{-1}\right)} \leq \int_{2^{l-2}}^{\infty} k^{-1 / \beta} d k=\gamma 2^{-\gamma(l-2)} .
$$


Write $i(p):=-p \log p$. Then for sufficiently large $N$,

$$
\begin{aligned}
& \sum_{l=N}^{\infty} i\left(P\left(L_{i}=l\right)\right) \\
& \quad \leq \sum_{l=N}^{\infty} i\left(\gamma 2^{-\gamma(l-2)}\right) \\
& \quad \leq \sum_{l=N}^{\infty} \gamma 2^{2 \gamma} 2^{-\gamma l}(-\log \gamma+\gamma l) \\
& \quad=\gamma 2^{2 \gamma}\left(\frac{2^{-\gamma N}}{1-2^{-\gamma}}(-\log \gamma)+\frac{2^{-\gamma(N+1)}+N 2^{-\gamma N}\left(1-2^{-\gamma}\right)}{\left(1-2^{-\gamma}\right)^{2}} \gamma\right) \\
& \quad \leq A(\gamma) N 2^{-\gamma N} .
\end{aligned}
$$

Let $M=n\left(L_{t}^{+}+\epsilon\right)$ and $N=\lceil n \epsilon / 2\rceil-1$. Then, for sufficiently large $n$,

$$
\begin{aligned}
P\left(S_{n}^{+c}\right) & H\left(L_{1} \mid S_{n}^{+c}\right) \\
& =P\left(S_{n}^{+c}\right) \sum_{l=0}^{\infty} i\left(\frac{P\left(L_{1}=l, \sum_{i=2}^{n} L_{i} \geq M-l\right)}{P\left(S_{n}^{+c}\right)}\right) \\
& =P\left(S_{n}^{+c}\right) \sum_{l=0}^{\infty} i\left(\frac{P\left(L_{1}=l\right) P\left(\sum_{i=2}^{n} L_{i} \geq M-l\right)}{P\left(S_{n}^{+c}\right)}\right) \\
\leq & \sum_{l=0}^{\infty} i\left(P\left(L_{1}=l\right) P\left(\sum_{i=2}^{n} L_{i} \geq M-l\right)\right) \\
\leq & \sum_{l=0}^{N-1} i\left(P\left(\sum_{i=2}^{n} L_{i} \geq M-l\right)\right)+\sum_{l=N}^{\infty} i\left(P\left(L_{1}=l\right)\right) \\
\leq & N i\left(P\left(\sum_{i=2}^{n} L_{i} \geq M-N+1\right)\right)+\sum_{l=N}^{\infty} i\left(P\left(L_{1}=l\right)\right) \\
\leq & \frac{n(n-1) t \epsilon^{2}}{2^{(n-1) t \epsilon / 2}}+\frac{n A(\gamma) \epsilon}{2^{(n-1) \gamma \epsilon / 2}} \leq \frac{1}{2^{n s \epsilon}} .
\end{aligned}
$$

Analogously we obtain the claim for $T_{n}^{+c}$.

Now, define events

$$
\begin{aligned}
\bar{S}_{n}^{+} & :=\left(\sum_{i=1}^{n} \bar{L}_{i}<n\left(L_{t}^{+}+\epsilon\right)\right), \\
\bar{S}_{n}^{-} & :=\left(\sum_{i=1}^{n} \bar{L}_{i}>n\left(L_{t}^{-}-\epsilon\right)\right), \\
\bar{T}_{n}^{+} & :=\left(\sum_{i=-n+1}^{-1} \bar{L}_{i}<(n-1)\left(L_{t}^{+}+\epsilon\right)\right), \\
\bar{T}_{n}^{-} & :=\left(\sum_{i=-n+1}^{-1} \bar{L}_{i}>(n-1)\left(L_{t}^{-}-\epsilon\right)\right), \\
\bar{B} & :=\left(\bar{L}_{0} \leq l\right) .
\end{aligned}
$$


Lemma 5 For $m \geq n\left(L_{t}^{+}+\epsilon\right)+l$ we have

$$
\begin{aligned}
I\left(\bar{X}_{-n+1:-1} ; \bar{X}_{1: n} \mid \bar{S}_{n}^{+} \cap \bar{T}_{n}^{+}\right) & =I\left(\bar{X}_{-n+1:-1} ; \bar{X}_{1: n} \mid \bar{B} \cap \bar{S}_{n}^{+} \cap \bar{T}_{n}^{+}\right) \\
& \leq I\left(\bar{Y}_{-m+1: 0} ; \bar{Y}_{1: m} \mid \bar{B} \cap \bar{S}_{n}^{+} \cap \bar{T}_{n}^{+}\right)
\end{aligned}
$$

whereas for $m \leq(n-1)\left(L_{t}^{-}-\epsilon\right)$ we have

$$
I\left(\bar{Y}_{-m+1: 0} ; \bar{Y}_{1: m} \mid \bar{S}_{n}^{-} \cap \bar{T}_{n}^{-}\right) \leq I\left(\bar{X}_{-n+1: 0} ; \bar{X}_{0: n} \mid \bar{S}_{n}^{-} \cap \bar{T}_{n}^{-}\right) .
$$

Proof: The claims follow by equality (35) and conditional data processing inequality

$$
I\left(U^{\prime} ; V^{\prime} \mid C\right) \leq I(U ; V \mid C)
$$

which holds if equalities $U^{\prime}=g(U)$ and $V^{\prime}=h(V)$ are satisfied on $C$.

There is an additional fact that we shall use. Let $I_{C}$ be the indicator function of event $C$. Observe that

$$
\begin{aligned}
P(C) I(X ; Y \mid C) & \leq P(C) I(X ; Y \mid C)+P\left(C^{c}\right) I\left(X ; Y \mid C^{c}\right) \\
& =I\left(X ; Y \mid I_{C}\right)=I(X ; Y)-I\left(X ; Y ; I_{C}\right)
\end{aligned}
$$

where $\left|I\left(X ; Y ; I_{C}\right)\right| \leq H\left(I_{C}\right) \leq 1$ by the information diagram [26].

Proof of Proposition 5: Observe that

$$
\begin{aligned}
H\left(X_{1} \mid S_{n}^{+c}\right) & =H\left(X_{1} \mid L_{1}\right)+H\left(L_{1} \mid S_{n}^{+^{c}}\right) \\
& \leq H\left(X_{0}\right)+H\left(L_{1} \mid S_{n}^{+^{c}}\right), \\
H\left(X_{-1} \mid T_{n}^{+^{c}}\right) & =H\left(X_{-1} \mid L_{-1}\right)+H\left(L_{-1} \mid T_{n}^{+^{c}}\right) \\
& \leq H\left(X_{0}\right)+H\left(L_{-1} \mid T_{n}^{+c}\right) .
\end{aligned}
$$

because $X_{1}$ is conditionally independent from $S_{n}^{+c}$ given $L_{1}$ and $X_{-1}$ is conditionally independent from $T_{n}^{+{ }^{c}}$ given $L_{-1}$. Now, assume that $n$ is sufficiently large so that bounds (48) and (49) hold true. For brevity, define events

$$
\begin{aligned}
C_{n}^{+} & :=T_{n}^{+} \cap S_{n}^{+}, \\
\bar{C}_{n}^{+} & :=\bar{T}_{n}^{+} \cap \bar{S}_{n}^{+} .
\end{aligned}
$$

Then inequalities (58), (59), (44), (46), (48), and (49) yield

$$
\begin{aligned}
P\left(C_{n}^{+^{c}}\right) I & \left(X_{-n+1:-1} ; X_{1: n} \mid C_{n}^{+^{c}}\right) \\
& \leq P\left(S_{n}^{+^{c}}\right) n H\left(X_{1} \mid{S_{n}^{+}}^{c}\right)+P\left(T_{n}^{+^{c}}\right)(n-1) H\left(X_{-1} \mid T_{n}^{+^{c}}\right) \\
& \leq \frac{2 n}{2^{(n-1) t \epsilon}} H\left(X_{0}\right)+\frac{2 n}{2^{(n-1) s \epsilon}} .
\end{aligned}
$$


Moreover, assume that $m \geq n\left(L_{t}^{+}+\epsilon\right)+l$. Then applying subsequently (57), (55), (36), (57), and (60) we obtain

$$
\begin{aligned}
E_{\bar{\nu}}(m) & =I\left(\bar{Y}_{-m+1: 0} ; \bar{Y}_{1: m}\right) \\
& \geq P\left(\bar{B} \cap \bar{C}_{n}^{+}\right) I\left(\bar{Y}_{-m+1: 0} ; \bar{Y}_{1: m} \mid \bar{B} \cap \bar{C}_{n}^{+}\right)-1 \\
& \geq P(\bar{B}) P\left(\bar{C}_{n}^{+}\right) I\left(\bar{X}_{-n+1:-1} ; \bar{X}_{1: n} \mid \bar{C}_{n}^{+}\right)-1 \\
& =P(\bar{B}) P\left(C_{n}^{+}\right) I\left(X_{-n+1:-1} ; X_{1: n} \mid C_{n}^{+}\right)-1 \\
& \geq P(\bar{B}) P\left(C_{n}^{+}\right)\left[I\left(X_{-n+1: 0} ; X_{1: n} \mid C_{n}^{+}\right)-H\left(X_{0} \mid C_{n}^{+}\right)\right]-1 \\
& \geq P(\bar{B}) P\left(C_{n}^{+}\right) I\left(X_{-n+1: 0} ; X_{1: n} \mid C_{n}^{+}\right)-H\left(X_{0}\right)-1 \\
& \geq P(\bar{B})\left[E_{\mu}(n)-1-P\left(C_{n}^{+c}\right) I\left(X_{-n+1: 0} ; X_{1: n} \mid C_{n}^{+c}\right)\right]-H\left(X_{0}\right)-1 \\
& \geq P(\bar{B}) E_{\mu}(n)-P\left(C_{n}^{+c}\right) I\left(X_{-n+1:-1} ; X_{1: n} \mid C_{n}^{+c}\right)-2 H\left(X_{0}\right)-2 \\
& \geq P(\bar{B}) E_{\mu}(n)-\left[\frac{2 n}{2^{(n-1) t \epsilon}}+2\right] H\left(X_{0}\right)-\frac{2 n}{2^{(n-1) s \epsilon}}-2 .
\end{aligned}
$$

Next, define events

$$
\begin{aligned}
& C_{n}^{-}:=T_{n}^{-} \cap S_{n}^{-}, \\
& \bar{C}_{n}^{-}:=\bar{T}_{n}^{-} \cap \bar{S}_{n}^{-} .
\end{aligned}
$$

By (45) and (47) we have

$$
\begin{aligned}
P\left(C_{n}^{-c}\right) & I\left(\bar{Y}_{-m+1: 0} ; \bar{Y}_{1: m} \mid C_{n}^{-c}\right) \\
& \leq\left(P\left(S_{n}^{-c}\right)+P\left(T_{n}^{-c}\right)\right) m \log 3 \leq \frac{2 m}{2^{(n-1) t \epsilon}} \log 3 .
\end{aligned}
$$

Assume that $m \leq(n-1)\left(L_{t}^{-}-\epsilon\right)$. Then applying subsequently (36), (57), (56), (57), and (62) we obtain

$$
\begin{aligned}
E_{\mu}(n) & =I\left(X_{-n+1: 0} ; X_{1: n}\right) \\
& \geq I\left(X_{-n+1:-1} ; X_{1: n}\right) \\
& =I\left(\bar{X}_{-n+1:-1} ; \bar{X}_{1: n}\right) \\
& \geq I\left(\bar{X}_{-n+1: 0} ; \bar{X}_{0: n}\right)-2 H\left(\bar{X}_{0}\right) \\
& \geq P\left(\bar{C}_{n}^{-}\right) I\left(\bar{X}_{-n+1: 0} ; \bar{X}_{0: n} \mid \bar{C}_{n}^{-}\right)-1-2 H\left(\bar{X}_{0}\right) \\
& \geq P\left(\bar{C}_{n}^{-}\right) I\left(\bar{Y}_{-m+1: 0} ; \bar{Y}_{1: m} \mid \bar{C}_{n}^{-}\right)-2 H\left(\bar{X}_{0}\right)-1 \\
& \geq E_{\bar{\nu}}(m)-1-P\left(C_{n}^{-c}\right) I\left(\bar{Y}_{-m+1: 0} ; \bar{Y}_{1: m} \mid C_{n}^{-c}\right)-2 H\left(\bar{X}_{0}\right)-1 \\
& \geq E_{\bar{\nu}}(m)-\frac{2 m}{2^{(n-1) t \epsilon}} \log 3-2 H\left(\bar{X}_{0}\right)-2 .
\end{aligned}
$$

From bounds (61) and (63) we obtain

$$
\begin{aligned}
& \frac{1}{\left[L_{t}^{-}-\epsilon\right]^{\beta}} \limsup _{n \rightarrow \infty} \frac{E_{\bar{\nu}}(n)}{n^{\beta}} \geq \limsup _{m \rightarrow \infty} \frac{E_{\bar{\nu}}(m)}{m^{\beta}} \geq \frac{P\left(\bar{L}_{0} \leq l\right)}{\left[L_{t}^{+}+\epsilon\right]^{\beta}} \limsup _{n \rightarrow \infty} \frac{E_{\bar{\nu}}(n)}{n^{\beta}}, \\
& \frac{1}{\left[L_{t}^{-}-\epsilon\right]^{\beta}} \liminf _{n \rightarrow \infty} \frac{E_{\bar{\nu}}(n)}{n^{\beta}} \geq \liminf _{m \rightarrow \infty} \frac{E_{\bar{\nu}}(m)}{m^{\beta}} \geq \frac{P\left(\bar{L}_{0} \leq l\right)}{\left[L_{t}^{+}+\epsilon\right]^{\beta}} \liminf _{n \rightarrow \infty} \frac{E_{\bar{\nu}}(n)}{n^{\beta}} .
\end{aligned}
$$

If we consider $t \rightarrow 0, \epsilon \rightarrow 0$, and $l \rightarrow \infty$ then the requested claims will follow by equation (39) and Proposition 3 , 


\section{A Mixing properties}

In this appendix we will discuss mixing properties of the generalized Santa Fe process. The setting makes use of the $L^{2}$ space of complex valued functions. Then, for a measure space $(\Omega, \mathcal{J}, \mu)$ let

$$
L_{0}^{2}(\Omega, \mathcal{J}, \mu):=\left\{f \in L^{2}(\Omega, \mathcal{J}, \mu): \int f d \mu=0\right\}
$$

and denote the inner product $(f, g)_{\mu}:=\int f \bar{g} d \mu$ and the norm $\|f\|_{\mu}:=\sqrt{(f, f)_{\mu}}$ for $f, g \in L^{2}(\Omega, \mathcal{J}, \mu)$. Let also $T: \Omega \rightarrow \Omega$ be an invertible transformation that preserves the measure, $\mu \circ T^{-1}=\mu$. The dynamical system $(\Omega, \mathcal{J}, \mu, T)$ is called mixing when $\lim _{n \rightarrow \infty}\left(f \circ T^{n}, g\right)_{\mu}=0$ for $f, g \in L_{0}^{2}(\Omega, \mathcal{J}, \mu)$. By the way, we know that any mixing dynamical system is ergodic [22, Chapter $1 . \S 6]$.

The following proposition generalizes Theorem 2 from [22, Chapter 10.\$1]. Whereas the original claim deals with finite direct products of dynamical systems, we will extend it here to infinite products. To the best of our knowledge this generalization has not been discussed in the literature so far. The proof is similar to the finite case, except for using a different orthonormal basis of the product space.

Proposition 6 Let $\left(\Omega_{j}, \mathcal{J}_{j}, \mu_{j}, T_{j}\right)$, where $j \in \mathbb{N}$, be dynamical systems with probability measures $\mu_{j}(\Omega)=1$. Consider the direct product $(\Omega, \mathcal{J}, \mu, T)$, where $\Omega=\times_{j=1}^{\infty} \Omega_{j}, \mathcal{J}=\otimes_{j=1}^{\infty} \mathcal{J}_{j}, \mu=\times_{j=1}^{\infty} \mu_{j}$, and $T(\omega)=\left(T_{j}\left(\omega_{j}\right)\right)_{j \in \mathbb{N}}$ for $\omega=$ $\left(\omega_{j}\right)_{j \in \mathbb{N}}, \omega_{j} \in \Omega_{j}$. If $\left(\Omega_{j}, \mathcal{J}_{j}, \mu_{j}, T_{j}\right)$ are mixing then $(\Omega, \mathcal{J}, \mu, T)$ is also mixing.

Proof: Let $\left(e_{\alpha_{j}, j}\right)_{\alpha_{j} \in A_{j}}$ be orthonormal bases of spaces $L^{2}\left(\Omega_{j}, \mathcal{J}_{j}, \mu_{j}\right)$ with $e_{0 j}=1$ and $e_{\alpha_{j}, j} \in L_{0}^{2}\left(\Omega_{j}, \mathcal{J}_{j}, \mu_{j}\right)$. Then the set

$$
\left\{e_{\emptyset}(\omega)=1\right\} \cup\left\{e_{\alpha}(\omega)=\prod_{j=1}^{k} e_{\alpha_{j}, j}\left(\omega_{j}\right)\right\}_{\alpha \in A_{1} \times A_{2} \times \ldots \times\left(A_{k} \backslash\{0\}\right), k=1,2, \ldots}
$$

with multi-indices $\alpha=\left(\alpha_{1}, \alpha_{2}, \ldots, \alpha_{k}\right)$ is an orthonormal basis of the space $L^{2}(\Omega, \mathcal{J}, \mu)$, cf., [27, page 29]. (Orthogonality of set (64) is obvious whereas its completeness follows from the completeness of the analogical orthonormal sets for finite products and the $L^{2}$-bounded martingale convergence.) Let $\alpha, \alpha^{\prime} \neq \emptyset$. We have $e_{\alpha}, e_{\alpha^{\prime}} \in L_{0}^{2}(\Omega, \mathcal{J}, \mu)$ and

$$
\left|\left(e_{\alpha} \circ T^{n}, e_{\alpha}^{\prime}\right)_{\mu}\right|=\prod_{j=1}^{k}\left|\left(e_{\alpha_{j}, j} \circ T_{j}^{n}, e_{\alpha_{j}^{\prime}, j}\right)_{\mu_{j}}\right| \leq\left|\left(e_{\alpha_{k}, k} \circ T_{k}^{n}, e_{\alpha_{k}^{\prime}, k}\right)_{\mu_{k}}\right|
$$

by Schwarz inequality if $\alpha$ and $\alpha$ have the same length $k$. Otherwise, $\left(e_{\alpha} \circ\right.$ $\left.T^{n}, e_{\alpha}^{\prime}\right)_{\mu}=0$. Hence $\lim _{n \rightarrow \infty}\left(e_{\alpha} \circ T^{n}, e_{\alpha}^{\prime}\right)_{\mu}=0$ holds by the hypothesis.

Any other functions $f, g \in L_{0}^{2}(\Omega, \mathcal{J}, \mu)$ can be represented as series $f=$ $\sum_{\alpha \neq \emptyset} f_{\alpha} e_{\alpha}$ and $g=\sum_{\alpha \neq \emptyset} g_{\alpha} e_{\alpha}$, where $\sum_{\alpha \neq \emptyset}\left|f_{\alpha}\right|^{2}, \sum_{\alpha \neq \emptyset}\left|g_{\alpha}\right|^{2}<\infty$. Assume without loss of generality that $\|f\|_{\mu}=\|g\|_{\mu}=1$. We will show that for every $\epsilon>0$, inequality $\left|\left(f \circ T^{n}, g\right)_{\mu}\right|<\epsilon$ holds for sufficiently large $n$. Let $F$ and $G$ be finite subsets of multi-indices such that $\left\|f-f^{\prime}\right\|_{\mu},\left\|g-g^{\prime}\right\|_{\mu}<\epsilon / 4$ for certain $f^{\prime}=\sum_{\alpha \in F} f_{\alpha}^{\prime} e_{\alpha}$ and $g^{\prime}=\sum_{\alpha \in G} g_{\alpha}^{\prime} e_{\alpha}$ where $f^{\prime}, g^{\prime} \in L_{0}^{2}(\Omega, \mathcal{J}, \mu)$ and 
$\left\|f^{\prime}\right\|_{\mu}=\left\|g^{\prime}\right\|_{\mu}=1$. For sufficiently large $n$, we have $\left.\mid\left(f^{\prime} \circ T^{n}, g^{\prime}\right)_{\mu}\right) \mid<\epsilon / 4$. Then

$$
\begin{aligned}
&\left.\mid\left(f \circ T^{n}, g\right)_{\mu}\right) \mid \leq\left.\left.\mid\left(f^{\prime} \circ T^{n}, g^{\prime}\right)_{\mu}\right)|+|\left(\left(f-f^{\prime}\right) \circ T^{n}, g^{\prime}\right)_{\mu}\right) \mid \\
&\left.\quad+\mid\left(f^{\prime} \circ T^{n},\left(g-g^{\prime}\right)\right)_{\mu}\right)|+|\left(\left(f-f^{\prime}\right) \circ T^{n},\left(g-g^{\prime}\right)_{\mu}\right) \mid \\
&<\epsilon / 4+\left\|f-f^{\prime}\right\|_{\mu}+\left\|g-g^{\prime}\right\|_{\mu}+\left\|f-f^{\prime}\right\|_{\mu}\left\|g-g^{\prime}\right\|_{\mu}<\epsilon,
\end{aligned}
$$

which completes the proof.

Now let us apply this result to the generalized Santa Fe process. A stochastic process $\left(X_{i}\right)_{i \in \mathbb{Z}}$ on $(\Omega, \mathcal{J}, P)$, where $X_{i}:(\Omega, \mathcal{J}) \rightarrow(\mathbb{X}, \mathcal{X})$, is called mixing if $\left(\mathbb{X}^{\mathbb{Z}}, \mathcal{X}^{\mathbb{Z}}, \mu, T\right)$ is mixing for $\mu=P\left(\left(X_{k}\right)_{k \in \mathbb{Z}} \in \cdot\right)$ and $T\left(\left(x_{i}\right)_{i \in \mathbb{Z}}\right)=\left(x_{i+1}\right)_{i \in \mathbb{Z}}$.

Proof of Proposition 2; Introduce an auxiliary process $\left(W_{i}\right)_{i \in \mathbb{Z}}$, where $W_{i}=\left(K_{i},\left(Z_{i k}\right)_{k \in \mathbb{N}}\right)$. Process $\left(W_{i}\right)_{i \in \mathbb{Z}}$ is a direct product of processes $\left(K_{i}\right)_{i \in \mathbb{Z}}$, $\left(Z_{i 1}\right)_{i \in \mathbb{Z}},\left(Z_{i 2}\right)_{i \in \mathbb{Z}}, \ldots$, which are all mixing for $p_{k} \in(0,1)$. Hence $\left(W_{i}\right)_{i \in \mathbb{Z}}$ is mixing by Proposition [6. (In our application, we take $\mu=P\left(\left(W_{i}\right)_{i \in \mathbb{Z}} \in \cdot\right.$ ), $\mu_{1}=P\left(\left(K_{i}\right)_{i \in \mathbb{Z}} \in \cdot\right)$, and $\mu_{k+1}=P\left(\left(Z_{i k}\right)_{i \in \mathbb{Z}} \in \cdot\right)$ for $k \geq 1$. The transformations are $T\left(\left(w_{i}\right)_{i \in \mathbb{Z}}\right)=\left(w_{i+1}\right)_{i \in \mathbb{Z}}, T_{1}\left(\left(k_{i}\right)_{i \in \mathbb{Z}}\right)=\left(k_{i+1}\right)_{i \in \mathbb{Z}}$, and $T_{k+1}\left(\left(z_{i}\right)_{i \in \mathbb{Z}}\right)=\left(z_{i+1}\right)_{i \in \mathbb{Z}}$ for $k \geq 1$.) Having established the mixing property for $\left(W_{i}\right)_{i \in \mathbb{Z}}$, we notice that $X_{i}=f\left(W_{i}\right)$ for a measurable function $f$. Hence $\left(X_{i}\right)_{i \in \mathbb{Z}}$ is mixing by Theorem 3 from 22 , Chapter $\left.10 . \S 1\right]$.

\section{Acknowledgment}

First, I would like to thank Richard Bradley for letting me know about his paper and Nicholas Travers for suggesting a few other references. Second, special thanks are due to Peter Grünwald for inviting me to Centrum Wiskunde \& Informatica, where the proof of Proposition 1 was drafted. Third, I appreciate comments of Jan Mielniczuk and the referees, regarding the paper composition.

\section{References}

[1] M. S. Pinsker, Information and Information Stability of Random Variables and Processes. Holden-Day, 1964.

[2] Ł. Dębowski, "A general definition of conditional information and its application to ergodic decomposition," Statist. Probab. Lett., vol. 79, pp. 12601268, 2009.

[3] J. P. Crutchfield and D. P. Feldman, "Regularities unseen, randomness observed: The entropy convergence hierarchy," Chaos, vol. 15, pp. 25-54, 2003.

[4] W. Hilberg, "Der bekannte Grenzwert der redundanzfreien Information in Texten — eine Fehlinterpretation der Shannonschen Experimente?" Frequenz, vol. 44, pp. 243-248, 1990.

[5] Ł. Dębowski, "On the vocabulary of grammar-based codes and the logical consistency of texts," IEEE Trans. Inform. Theor., vol. 57, pp. 4589-4599, 2011. 
[6] P. J. Brockwell and R. A. Davis, Time Series: Theory and Methods. Springer, 1987.

[7] F. L. Ramsey, "Characterization of the partial autocorrelation function," Ann. Statist., vol. 2, pp. 1296-1301, 1974.

[8] P. D. Finch, "On the covariance determinants of autoregressive and moving average models," Biometrika, vol. 47, pp. 194-211, 1960.

[9] T. M. Cover and J. A. Thomas, Elements of Information Theory. Wiley, 1991.

[10] U. Grenander and G. Szegő, Toeplitz Forms and Their Applications. Berkeley: University of California Press, 1958.

[11] P. D. Grünwald, The Minimum Description Length Principle. The MIT Press, 2007.

[12] T. Gramss, "Entropy of the symbolic sequence for critical circle maps," Phys. Rev. E, vol. 50, pp. 2616-2620, 1994.

[13] J. P. Crutchfield and K. Young, "Inferring statistical complexity," Phys. Rev. Lett., vol. 63, pp. 105-108, 1989.

[14] W. Ebeling, "Prediction and entropy of nonlinear dynamical systems and symbolic sequences with LRO," Physica D, vol. 109, pp. 42-45, 1997.

[15] R. C. Bradley, "On the strong mixing and weak Bernoulli conditions," $Z$. Wahrsch. verw. Geb., vol. 50, pp. 49-54, 1980.

[16] Štefan Šujan, "Ergodic theory, entropy, and coding problems," Kybernetika, vol. 19, pp. 3-66, 1983.

[17] R. C. Bradley, Introduction to Strong Mixing Conditions. Heber City: Kendrick Press, 2007.

[18] C. R. Shalizi and J. P. Crutchfield, "Computational mechanics: Pattern and prediction, structure and simplicity," J. Statist. Phys., vol. 104, pp. 817-879, 2001.

[19] N. F. Travers and J. P. Crutchfield, "Infinite excess entropy processes with countable-state generators," http://arxiv.org/abs/1111.3393

[20] C. G. de Marcken, "Unsupervised language acquisition," Ph.D. dissertation, Massachussetts Institute of Technology, 1996.

[21] Ł. Dębowski, "Variable-length coding of two-sided asymptotically mean stationary measures," J. Theor. Probab., vol. 23, pp. 237-256, 2010.

[22] I. P. Cornfeld, S. V. Fomin, and Y. G. Sinai, Ergodic Theory. Springer, 1982.

[23] G. Cariolaro and G. Pierobon, "Stationary symbol sequences from variablelength word sequences," IEEE Trans. Inform. Theor., vol. 23, pp. 243-253, 1977. 
[24] R. M. Gray and J. C. Kieffer, "Asymptotically mean stationary measures," Ann. Probab., vol. 8, pp. 962-973, 1980.

[25] R. Timo, K. Blackmore, and L. Hanlen, "On the entropy rate of wordvalued sources," in Proceedings of the Telecommunication Networks and Applications Conference, ATNAC 2007, 2007, pp. 377-382.

[26] R. W. Yeung, First Course in Information Theory. Kluwer Academic Publishers, 2002.

[27] Y. M. Berezanskiı̌, Selfadjoint Operators in Spaces of Functions of Infinitely Many Variables. American Mathematical Society, 1986. 